\title{
Semantic Texton Forests for Image Categorization and Segmentation
}

\author{
Miss Nilophar Mullani ${ }^{1}$, Prof. Arati Dandavate ${ }^{2}$ \\ PG Student, Computer Department, JSPM's JSCOE, Handewadi, Pune ${ }^{1}$ \\ Associate Professor, Computer Department, JSPM's JSCOE, Handewadi, Pune ${ }^{2}$
}

\begin{abstract}
The Semantic Texton Forest (STF) is a proficient and amazing low-level element which can be successfully utilized in the semantic division of pictures. STF's are connected to neighborhood space-time volumes as an amazing discriminative codebook. Since STFs act legitimately on video pixels without utilizing costly descriptors, visual codeword age by STFs is incredibly quick. Semantic division is an undertaking of synchronous item division and acknowledgment. The semantic division, can be thought as an expansion of the well-known scene characterization issue where the element to arrange isn't any longer the entire picture yet single gathering of pixels. The pack of semantic textons consolidates a histogram of semantic textons over a picture locale with a district earlier class circulation. The fundamental commitment is a picture level earlier for division that underlines those classifications that the programmed order accepts to be available. The primary utilization of Random Forests is that distinctive component types can without much of a stretch be consolidated bringing about an increasingly broad classifier.
\end{abstract}

Keywords: Semantic Texton Forest, Bag of Textons, Random Forest, Semantic Segmentation

\section{INTRODUCTION}

Segmentation is fundamental for picture investigation assignments. Semantic division depicts the way toward partner every pixel of a picture with a class name, (for example, blossom, individual, street, sky, sea, or vehicle). Semantic division is one of the abnormal state assignments that make ready towards complete scene understanding. With the prominence of profound learning as of late, numerous semantic division issues are being handled utilizing profound designs, regularly Convolutional Neural Nets, which outperform different methodologies by a vast edge as far as precision and proficiency. Additionally, we will anticipate and deal with how we trust this item will be utilized so as to pick up a superior comprehension of the venture, diagram ideas that might be grown later, and report thoughts that are being considered, however might be disposed of as the item creates.

It depicts [1] a methodology for semantic division of pictures. Two tale surface highlights dependent on DCT information are presented in the Semantic Texton Forest system. The viability of the proposed semantic division framework has been exhibited by contrasting it and the other cutting edge approaches.

They have displayed [2] methods for feebly regulated learning for semantic division. They gave this undertaking a role as a MIL issue. Additionally utilized the outside dataset for geometric setting estimation to additionally regularize arrangement. They present two methods that enable us to tackle this issue. First is worried about the likelihood assesses in the leaf hubs of the forest. The second improves the structure of the forest by methods for perform Multiple Tasks Learning (MTL). Look at [3] semantic texton forests, and assess their utilization for picture classification and semantic division. Semantic Texton Forests (STFs) exhibit that one can fabricate amazing texton codebooks without registering costly channel banks, and without showing exorbitant k-implies grouping and closest neighbor task.

We will really expound diverse arrangement calculations. We utilize the standard train/test parts, and the hand-marked ground truth to prepare the classifiers. Image classification execution is estimated as mean normal exactness. Division execution is estimated as both the class normal precision and the worldwide accuracy.

\section{RELATED WORK}

G. M. Run et al. [4] has vision following applications can give ongoing on location data utilizing position data of undertaking related elements. Mechanized acknowledgment of the undertaking related elements on a construction site is imperative to deliver ongoing consequences of following. Randomized decision forest is a classifier that compares of numerous choice trees. A class circulation is doled out to every hub in the paired tree, which speaks to the likelihood of a class given that the procedure achieves the hub in a tree. It very well may be inferred that Semantic Texton Forests can possibly consequently perceive items to be followed on a site if proper preparing and testing is given. 
H. Lombaert et al. [5] represent the principal contrasts between standard Random Decision Forests (RF) and Laplacian Forests (LF), which basically live in I) how pictures are chosen through guided sacking amid preparing, and II) how decision trees are weighted amid testing. Here, the Laplacian eigenmap was constructed utilizing a basic fondness measure, in view of the total of squared contrasts of pictures; yet watch an improvement in precision. This is another, effective and exact procedure for the semantic division of medical pictures.

A. Montillo et al. [6] proposed the Entangled Decision Forest (EDF) as another discriminative classifier which accomplishes higher expectation precision and abbreviated choice time. This spreads information starting with one piece of the timberland then onto the next which speeds learning, improves classifier speculation and catches long range-semantic setting. They propose infusing arbitrariness in a guided manner, in which hub highlight types and parameters are haphazardly drawn from an educated (non-uniform) conveyance.

C. Keskin et al. [7] bunched the expansive preparing sets utilizing ghastly grouping and prepared master Part Classification Forests (PCFs) on each group. Thus, we partitioned the intricate issue into less complex sub issues and prepared specialists. The techniques keep running in real- time on the CPU, and can be ported to the GPU for further increment in speed. This is a binary bunching technique, which can be analyzed to progressively group information into numerous groups. For the hand shape acknowledgment issue, shape classification forest(SCF) is proposed as a successful arrangement, which is shown on an expansive ASL dataset.

S. Battiato et al. [8] proposes a strategy to perceive scene classes utilizing packs of visual words acquired progressively dividing into sub-region the info pictures. The proposed technique has indicated promising outcomes in spite of to the way that no intricate learning system has been utilized. Each picture pixel is then related to the nearest Texton considering its channel bank reactions. They propose to increase the fundamental pack of visual words portrayal consolidating it with a various leveled parceling of the picture.

F. Perronnin et al. [9] acquainted a novel methodology with picture classification which comprises in applying the Fisher part structure on a visual vocabulary. They propose this structure to picture arrangement where the data signals are pictures and where the central generative model is a visual vocabulary. This is finished by taking the angle of the log-probability concerning a subset of the parameters.

A. Mojsilovic et al. [10] was tried on an expansive number of medicinal pictures accumulated on the WWW. Their outcomes show that exact arrangement. They propose a semantically based arrangement of visual highlights, their significance and association for catching the semantics of various imaging modalities. The principle objective is to structure a list of capabilities that is connected to human impression of picture content. Such a set considers an effective classification metric, just as natural questions.

\section{PROPOSED ALGORITHM}

\section{Description of the Proposed Algorithm:}

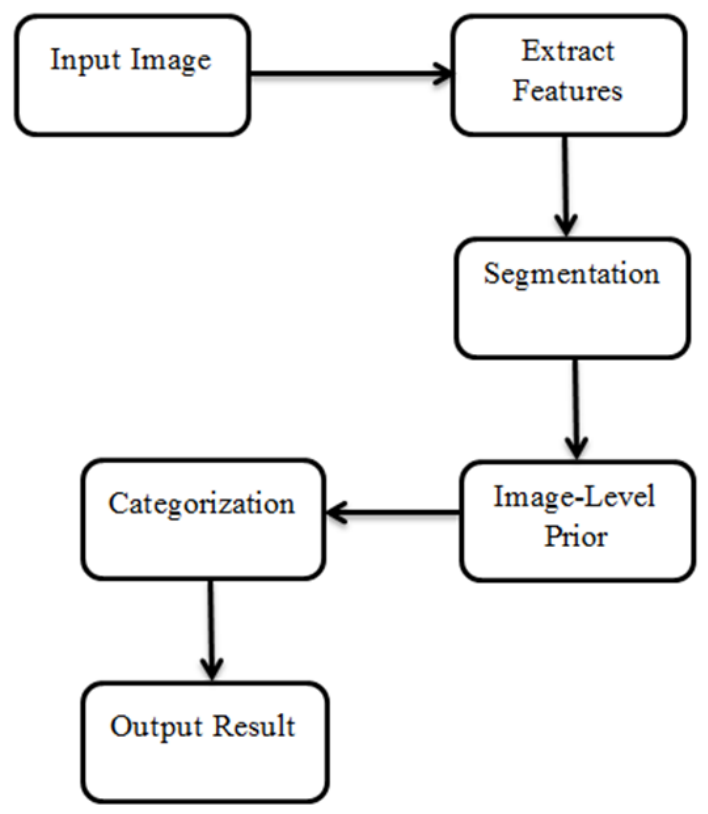

Fig. 1 Proposed system 
We will take a gander at two utilizations of STFs: picture arrangement (deducing the article classifications present in a picture) and semantic division (isolating the picture into intelligible areas and all the while sorting every locale). The instrument that will be utilized for both of these is the sack of semantic textons. This is registered over a given picture district, and expands the sack of words demonstrate by joining a progressive histogram of the semantic textons with an earlier classification appropriation. In the event that the dataset is marked it goes under a directed issue, it the dataset is unlabelled then it is an unsupervised issue. In this application we are going to use an unsupervised data for image segmentation.The K-infers estimation is an iterative strategy that is used to fragment an image into K groups.

The K-implies grouping calculation utilizes iterative refinement to deliver a last outcome. The calculation inputs are the quantity of bunches $\mathrm{K}$ and the informational collection. By thinking about the picture as a whole, a profoundly discriminative descriptor for arrangement can be gotten. For division, a sack of semantic textons can be figured for some nearby rectangular areas and afterward a second randomized decision forest can be assembled which accomplishes productive and exact division by illustration on appearance and semantic setting. The division calculation relies upon picture data that even with semi neighborhood setting can regularly be equivocal. The worldwide measurements of the picture, in any case, can be increasingly discriminative and might be adequate to precisely evaluate the picture order. It is subsequently helpful to utilize arrangement as an image level prior to improve division by underscoring the classifications destined to be available.

\section{PSEUDO CODE}

The K-means clustering algorithm:

Step 1) Initialization

Step 2) Load the image

Step 3) Separate R,G,B channel

Step 4) Apply Local histogram equalization to each channel

Step 5) Apply K-means clustering algorithm

Step 6) Update the center and cluster the image until it satisfy the condition

Step 7) Categorization

Step 8) Reshape the cluster into output image

Step 9) End

\section{SIMULATION RESULTS}

Local histogram equalization technique required initialization of the window size. By varying the size of the window, we can check for better output image. Generally more is the size of the window, better will the output image. First we have taken the window size of 50x50. Then we check the output again by taking the window size of $75 \times 75,100 \times 100$ and 150x150. But with the increase in the size of the window, the computation time will increase. So to increase the quality of the output image by increasing the window size will cost computation time. So one have to decide between the computation time and quality of the image based on the type of the application.Again we need to initialize number of cluster $\mathrm{k}$ and center of each R,Gand B channel. So these values are randomly initialized. We have taken $\mathrm{k}=2$. The proposed strategy is contrasted and k-implies, fuzzy c-implies and subtractive bunching technique. Fig below shows segmentation of input image $\&$ then further processed on that.

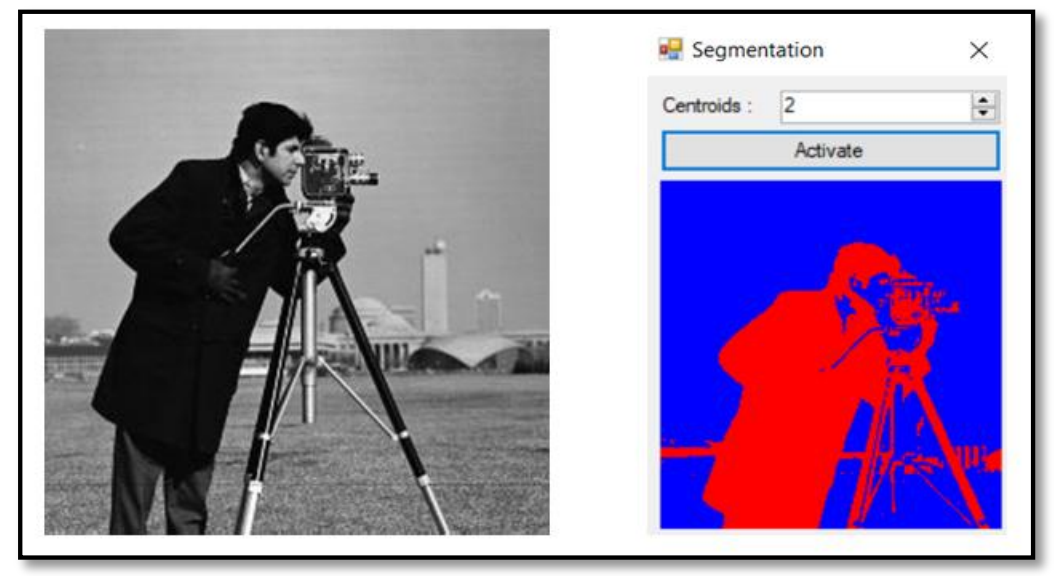

Fig.2. Performance analysis 


\section{CONCLUSION AND FUTURE WORK}

We are concentrating a model for Semantic Segmentation of the picture in various classes of articles. Division intends to name distinctive associated areas of the picture as having a place with explicit item class. The application proposed a technique for shading picture division utilizing k-implies bunching calculation. We have used local histogram equalization method to adapt the contrast based on the input image. Our primary target is recover explicit class pictures which would help in better preparing of the dataset. The primary utilization of Random Forests is that distinctive component types can without much of a stretch be joined bringing about a progressively broad classifier. Semantic texton forests (STFs) are randomized decision forest utilized for both bunching and characterization. Here we utilized MSRC Datasets which contain 21 unique classes labeled database. The framework will be prepared on set of pictures of some limited classes of items. We in this way explore how order can go about as a picture level before improve division by underlining the classifications well on the way to be available. They are incredibly quick to both train and test, particularly contrasted and k-implies grouping and closest neighbor task of highlight descriptors. In the future, we can introduce a new and better method of local histogram equalization to improve the computation time. Again more efficient method of histogram equalization base method can be approach in the future. The initial value of number of cluster and initial center plays an important role, so we can try in the future to find the number of cluster and initial center using the information from the histogram.

\section{REFERENCES}

[1]. D. Ravì, M. Boberb, G. M. Farinellaa, M. Guarnerac and S. Battiato, "Semantic Segmentation of Images Exploiting DCT Based Features and Random Forest," Pattern Recognition in 2015

[2]. A. Vezhnevets and J. M. Buhmann, “Towards Weakly Supervised Semantic Segmentation by Means of Multiple Instance and Multitask Learning,"2010 IEEE Conference on Computer Vision and Pattern Recognition in 2010.

[3]. Matthew Johnson and Jamie Shotton, "Semantic Texton Forests," based on the presentations at the international computer vision summer school - ICVSS (pp.173-203) in 2010.

[4]. G. M. Jog, M.-W. Park, and I. Brilakis, "Truck-Face Recognition using Semantic Texton Forests,"9th Construction Specialty Conference, in June 2011.

[5]. H. Lombaert, D. Zikic, A. Criminisi, and N. Ayache, "Laplacian Forests: Semantic Image Segmentation by Guided Bagging," International Conference on Medical Image Computing in 2014.

[6]. A. Montillo, J. Shotton, J. Winn and J. E. Iglesias, "Entangled Decision Forests and Their Application for Semantic Segmentation of CT Images," Information Processing in Medical Imaging pp. 184-196, 2011.

[7]. C. Keskin, F. Kırac, Y. E. Kara, and L. Akarun, "Hand Pose Estimation and Hand Shape Classification using Multilayered Randomized Decision Forests," Computer Vision - ECCV 2012 pp. 852-863, 2012.

[8]. S. Battiato, G. M. Farinella, G. Gallo and D. Ravi, "Scene Categorization Using Bag Of Textons On Spatial Hierarchy," IEEE International Conference on Image Processing in 2008.

[9]. F. Perronninand C. Dance, "Fisher Kernel son Visual Vocabularies for Image Categorization," IEEE Conference on Computer Vision and Pattern Recognition, 2007.

[10]. A. Mojsilovic and J. Gomes, "Semantic Based Categorization, Browsing and Retrieval In medical Image Databases," International Conference on Image Processing, 2002. 